www.jmscr.igmpublication.org

Impact Factor 5.84

Index Copernicus Value: 83.27

ISSN (e)-2347-176x ISSN (p) 2455-0450

crossref DOI: _https://dx.doi.org/10.18535/jmscr/v5i7.20

Journal Of Medical Science And Clinical Research

\title{
Correlation between Serum Homocysteine Levels and Serum Vitamin B12 Levels in First Time Acute MI Patients with Relative Lack of Conventional Risk Factors in North Indian (Rural) Population
}

\author{
Authors \\ Dr Hariharan Munganda, Dr Rishi Gupta, Dr Subrat Akhoury, Dr Simmimanocha, \\ Dr Umeshkohli, Dr Dyaneshamle \\ Asian Institute of Medical Sciences, Faridabad, New Delhi, India
}

\section{Introduction}

Indian patients have higher incidence of cardiac diseases inspite of the conventional risk factors comparable to western counterpart. The increased excess cardiac risk in Indians as compared to western population remains unexplained. Low serum vitamin B 12 and high serum homocysteine levels are known predictors of cardiovascular mortality and are highly frequent in Indians .Our present study was taken to ascertain whether these two factors are responsible for excess cardiac risk in indians.

\section{Aim of the study}

We in our study aimed to study subjects with AMI in whom most of the known risk factors for cardiovascular disease are absent but were deficient in vitamin B12 or having high $\mathrm{S}$. homocysteine levels and correlate the two parameters.

\section{Material and Methods}

Subjects presenting to our Asian tertiary care hospital who were having first time MI (naive) patients without any cardiac disease or any treatment and without any family history. All patients with STEMI less than 12hours of duration and no past history of cardiac disease/DM/HTN/ Dyslipidemia were included. After obtaining written informed consent, detailed history was sought and subjects were again evaluated for routine laboratory biochemical parameters including Serum vitamin B12 levels and serum homocysteine levels, coronary angiography and ECHO. 100 Subjects who were found to be suffering from AMI were included as cases, and 20 subjects showing symptoms of chest pain but lacking any elevation in $\mathrm{CK}$ total or CK MB, no changes on ECG and normal ECHO and angiographic findings were included as controls.

\section{Results}

Various risk factors of AMI including Age, non vegetarian diet, DM, HTN, Family history, BMI, Dyslipidemia, Low HDL, High TG, LDL, Cholesterol were found to be lower in cases compared to controls. Serum vitamin B 12 levels were found to be significantly lower $(<0.0001)$ and serum homocysteine levels $(<0.0001)$ were significantly higher in cases. Further Weak downhill ( $\mathrm{r}=-0.367)$ but significant correlation was noted between $\mathrm{S}$. Vit B 12 and $\mathrm{S}$. 
Homocystein levels. Also significant association was noted between low s. Vit. B12 and high homocysteine and Acute MI. ROC curve was plotted to evaluate predictive value of parameters for AMI. Vitamin B12 was found to have AUC of
$96.2 \%$ and sensitivity of $95 \%$ and specificity of $100 \%$ at cut off value lower than $360 \mathrm{pg} / \mathrm{L}$. Also Serum homocysteine was found to have AUC of $93.2 \%$ and sensitivity of $86 \%$ and specificity of $100 \%$ at cut off value higher than $25.3 \mathrm{mcg} / \mathrm{L}$.

Table: General characteristics of study subjects

\begin{tabular}{llccc}
\hline Characteristics & & Controls & Cases & P value \\
\hline Age (years) & & $52.5 \pm 8.9$ & $46.5 \pm 16.21$ & 0.11 \\
Gender & Male & $6(30)$ & $88(88)$ & $<0.0001$ \\
& Female & $14(70)$ & $12(12)$ & 0.05 \\
Diet & Vegetarian & $9(45)$ & $68(68)$ & 0.010 \\
& Non-Vegetarian & $11(55)$ & $32(32)$ & 0.040 \\
Alcohol & & $6(30)$ & $9(9)$ & $<0.0001$ \\
Smoking & & $6(30)$ & $12(12)$ & $<0.0001$ \\
DM & & $6(6)$ & 0.009 \\
HTN & & $12(60)$ & $10(10)$ & $<0.0001$ \\
BMI (Kg/m $\left.{ }^{2}\right)$ & $9(45)$ & $24.8 \pm 2.5$ & 0.810 \\
Dysliipidemia & & $14(14)$ & 0.19 \\
Family H/O & & $6(30)$ & $13(13)$ & 0.77 \\
PT/INR & Deranged & $3(15)$ & $8(8)$ & 0.083 \\
S. HDL(mg/dl) & & $0(0)$ & $44.7 \pm 13.2$ & 0.008 \\
S. Chol(mg/dl) & & $43.8 \pm 12.5$ & $172.5 \pm 29.3$ & 0.900 \\
S. LDL(mg/dl) & & $184.4 \pm 17.3$ & $117.3 \pm 28.3$ & $<0.0001$ \\
S. TG (mg/dl) & $135.2 \pm 19.0$ & $139.7 \pm 63.4$ & $<0.0001$ \\
CK (U/L) & & $141.6 \pm 42.2$ & $112.8 \pm 1075.4$ & $<0.0001$ \\
CK MB (ng/ml) & & $189.8 \pm 78.8$ & $264.2 \pm 211.7$ & $<0.0001$ \\
Vit B12 (ng/ml) & & $30.2 \pm 6.9$ & $205.1 \pm 173.5$ & $<0.0001$ \\
Homocystein (mcg/L) & & $629.0 \pm 207.9$ & $64.02 \pm 15.7$ & \\
Coronary & SVD & $14.9 \pm 4.8$ & $19(19)$ & 0.021 \\
angiography findings & DVD & $0(0)$ & $14(14)$ & \\
& TVD & $0(0)$ & 45.19 .2 & \\
Ejection fraction on ECHO (\%) & $0(0)$ & &
\end{tabular}

Table: Association of serum vitamin B12 levels with AMI

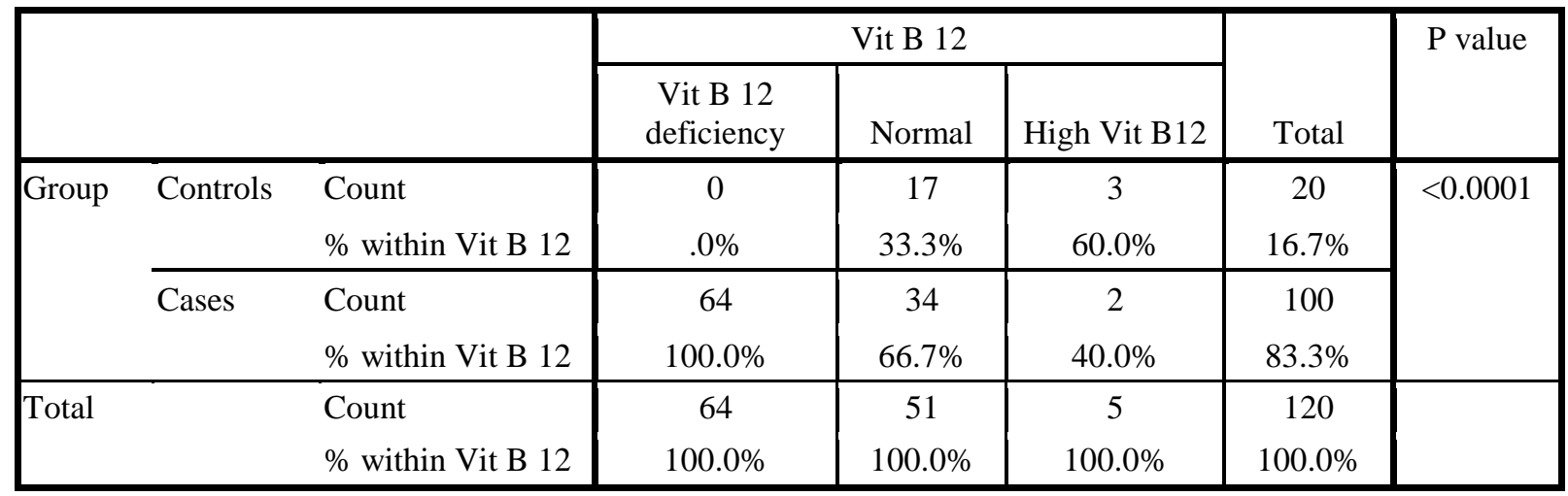

\begin{tabular}{|l|l|l|l|}
\hline ODDS Ratio & \multicolumn{2}{|c|}{ 95\%C.I. } & P value \\
\hline 72.45 & 4.25 & 1233.49 & 0.0031 \\
\hline
\end{tabular}


Table: Correlation of serum vitamin B12 with Serum Homocysteine

\begin{tabular}{|l|c|c|c|}
\hline & & $\mathrm{r}$ & $\mathrm{p}$ Value \\
\hline Vit. B 12 & S. Homocysteine & -0.367 & $<0.0001$ \\
\hline
\end{tabular}

Table: Association of Serum Homocysteine levels with AMI

\begin{tabular}{|c|c|c|c|c|c|}
\hline & \multicolumn{2}{|c|}{ S. Homocystein $(\mathrm{mcg} / \mathrm{L})$} & \multirow[b]{2}{*}{ Total } & \multirow[t]{2}{*}{$P$ value } \\
\hline & & Normal & Hyperhomocysteinurea & & \\
\hline \multicolumn{2}{|c|}{ Group Controls Count } & 17 & 3 & 20 & \multirow[t]{4}{*}{$<0.0001$} \\
\hline & $\%$ within S. Homocysteine $(\mathrm{mcg} / \mathrm{L})$ & $65.4 \%$ & $3.2 \%$ & $16.7 \%$ & \\
\hline \multirow[t]{2}{*}{ Cases } & Count & 9 & 91 & 100 & \\
\hline & $\%$ within S. Homocysteine (mcg/L) & $34.6 \%$ & $96.8 \%$ & $83.3 \%$ & \\
\hline \multirow[t]{2}{*}{ Total } & Count & 26 & 94 & 120 & \\
\hline & $\%$ within S. Homocysteine $(\mathrm{mcg} / \mathrm{L})$ & $100.0 \%$ & $100.0 \%$ & $100.0 \%$ & \\
\hline
\end{tabular}

\begin{tabular}{|l|c|c|c|}
\hline ODDS Ratio & \multicolumn{2}{|c|}{$95 \%$ C.I. } & P value \\
\hline 57.29 & 14.05 & 233.64 & $<0.0001$ \\
\hline
\end{tabular}

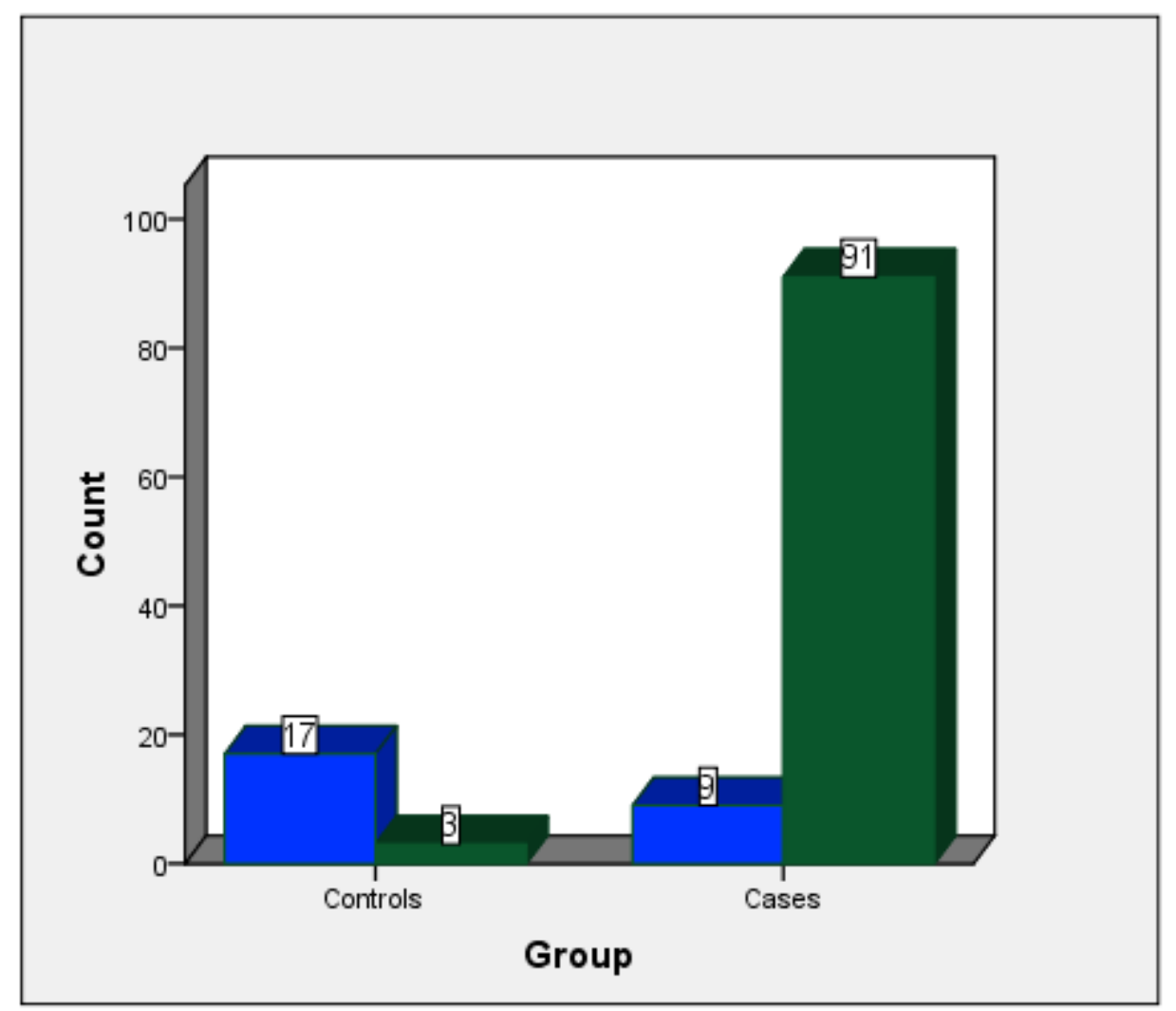

S. Homocystein $(\mathrm{mcg} / \mathrm{L})$

Normal

Hyperhomocysteinurea

Table: Predictive significance of Vit B 12 and Homocysteine for AMI

\begin{tabular}{|l|c|c|c|c|c|c|c|c|}
\hline Parameter & & \multirow{2}{*}{} & \multirow{2}{*}{} & \multicolumn{2}{|c|}{ Asymptotic 95\% Confidence Interval } & Cut-off & Sensitivity & Specificity \\
\cline { 5 - 6 } & Area & Std. Error & P value & Lower Bound & Upper Bound & & & \\
\hline Vit . B12 & .962 & .017 & .000 & .928 & .996 & 360.0 & $95 \%$ & $100 \%$ \\
\hline S. homocysteine & .932 & .023 & .000 & .887 & .977 & 25.3 & $86 \%$ & $100 \%$ \\
\hline
\end{tabular}




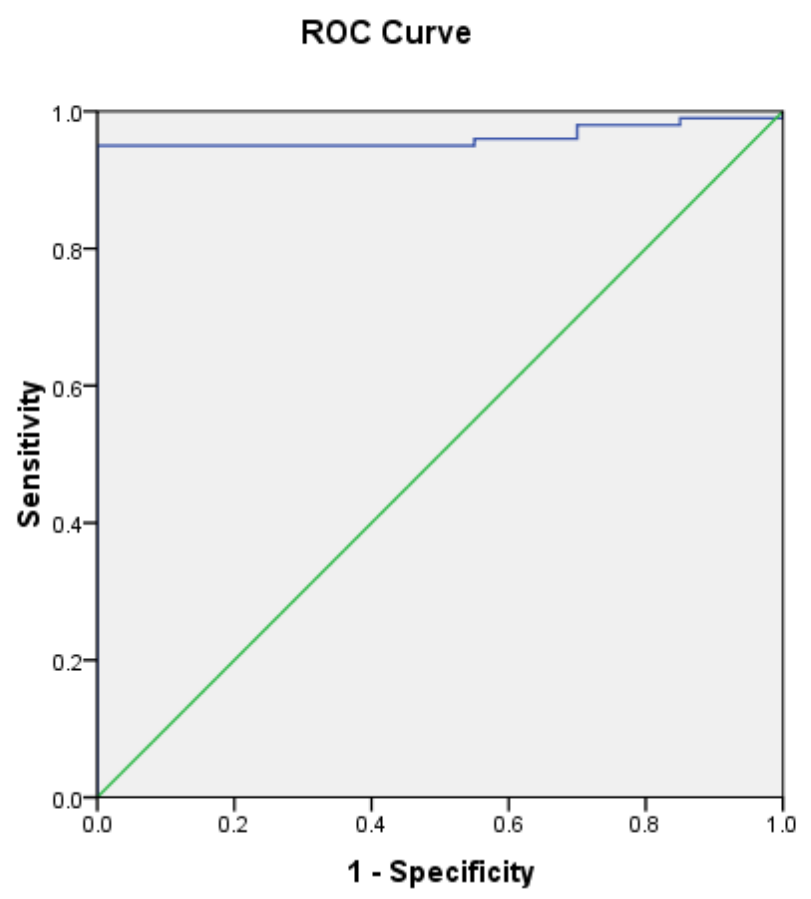

ROC curve for S. Vit B12

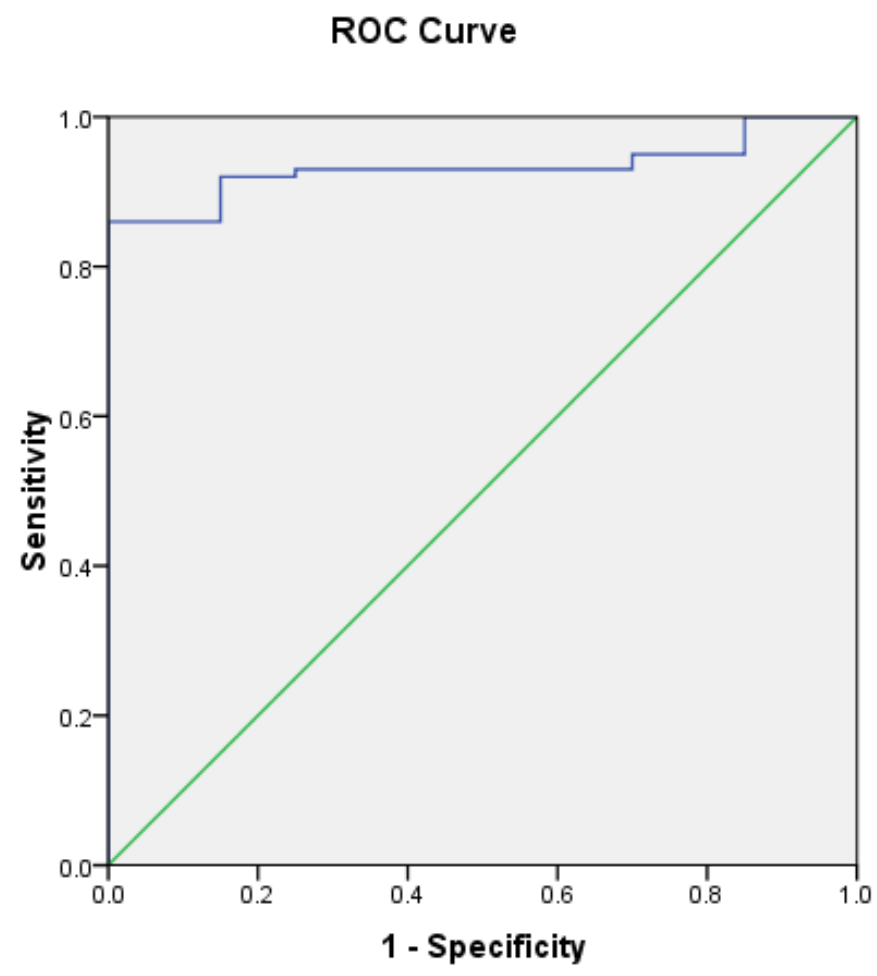

ROC curve for S. Homocysteine. 


\section{Conclusion}

In individuals especially younger age group(2030years) who lack conventional risk factors/ family history of coronary artery disease, low serum vitamin B12 levels and high serum homocysteine levels pose significant risk of heart disease. Serum vitamin B12 and serum homocysteine levels should be regularly assessed to predict the risk of AMI in subjects with lack of other risk factors along with dietary and lifestyle changes.

Cardiovascular disease has been associated with both homocysteine and vitamin B12 levels [1-6]. Elevated homocysteine levels are associated with increased thrombosis andincreased extent of myocardial injury. Very high levels of homocysteine have been previously reported in healthy adult populations in Asia especially India with nutritional deficiency and lead pollution being possibly the major determinants.

Homocysteine is a thiol-containing amino acid derived from the demethylation of methionine that circulates in plasma in 3 forms: as a single free amino acid (1\%), as homocysteine or cysteinehomocysteinedisulfides (20\% to $30 \%$ ), or bound to plasma proteins $(70 \%$ to $80 \%)$. Together, these account for total plasma homocysteine (tHcy). Inborn errors of metabolism arising from a deficiency of Hcy-metabolizing enzymes result in extremely high tHcy concentrations (severe hyperhomocysteinemia) and are associated with prematurevascular thrombosis, possibly as a result of oxidative damage mediated by the sulfhydryl group of free single-chain homocystine. Mild hyperhomocysteinemia results from both nutritional and more subtle genetic influences. ${ }^{[1-7]}$ Theiris evidence from an unexplained observation that although homozygosity for the $\mathrm{T}$ allele of the C677T polymorphism of methylene tetrahydrofolatereductase is associated with mild hyperhomocysteinemia, studies have failed to demonstrate this genotype as a risk factor for MI and stroke. In addition, obligate heterozygotes for cystathionine $\beta$ synthase deficiency (ie, parents of children with homocystinuria) do not exhibit evidence of carotid or femoral atherosclerosis despite elevated tHcy levels. This lack of association between genetic abnormalities resulting in mild hyperhomocysteinemia and arterial disease further weakens the hypothesis that mild elevations in tHcy directly promote atherosclerosis and thrombosis. ${ }^{\text {[24] }}$

Vitamin B12 is a water-soluble vitamin needed for normal nerve cell activity, DNA replication, and production of the mood-affecting substance SAMe (S-adenosyl-L-methionine). Vitamin B12 acts with folic acid and vitamin B6 to control homocysteine levels. Low serum vitamin B12 concentrations are common in elderly patients in the general population. Humans are dependent on dietary intake of vitamin B12, mainly from animal food products, such as meat, fish and dairy products. Vitamin B12 deficiency may lead to a wide range of complications, of which megaloblastic anaemia is the most common. Absorption of vitamin B12 is a complex process, in which the stomach plays an important role. First, digesting enzymes liberate vitamin B12 from its binding protein. Free vitamin B12 subsequently binds to intrinsic factor, produced by parietal cells, to form an IF-B12-complex. Only the IF-B12-complex can be absorbed in the terminal ileum. Because of the complexity of this absorption process, there are several causes of vitamin B12 deficiency, such as low dietary intake, low production of protein digesting enzymes, low production of intrinsic factor, as a result of atrophic gastritis (whether or not induced by an infection with Helicobacter pylori) or impaired absorption by ileal pathology, respectively. ${ }^{[1-2]}$

Some conclude that low vitamin B12 and folic acid concentrations are associated with an increased risk of stroke, and the relationship for vitamin B12 is independent from the other known modifiable stroke risk factors. For understanding the effects of B12 and folate in stroke patients, more detailed follow-up studies with long period are needed. ${ }^{[12]}$ Hyperhomocysteinemia is an independent risk factor for atherothrombotic 
cerebral stroke. Vitamin B12 and folic acid are important determinants of homocysteine metabolism. ${ }^{[12]}$

Subclinical deficiencies of folate, vitamins B12 and B6, and inheritance of the thermolabile variant of methylenetetra hydrofolatereductase are associated with modest elevations of homocysteineabove the 90th percentile of the normal range. Other conditions, including renal impairment, hypothyroidism, and drug therapy (eg, folate antimetabolites, theophylline, smoking, or oral contraceptives), are also associated with mild hyperhomocysteinemia. Whether this can cause premature arterial thrombosis is a contentious issue. Retrospective case-control studies have associated raised total homocysteine (tHcy) with both arterial and venous thrombosis.In contrast, results from prospective studies have been inconsistent, both supporting and refuting raised tHcy levels as a risk factor for myocardial infarction (MI). ${ }^{[1-6]}$

Normal serum Hcy levels might correspond to patients with no previous ischemic heart disease, whose myocardial cells are not already affected. The rise in Hcy levels on days after the infarction would then be explained by the post-infarction presence of a "frontier area" of cardiac cells between infarcted and healthy tissues. It may be that these cells are not necrosed but remain in a situation of ischemia (hypoxemia), with a reduced capacity to metabolize Hcy. The pathophysiological mechanism by which risk increases is not clearly understood but includes such aspects as a toxic effect on the vascular endothelium, impaired endothelium-dependent relaxation, a hypercoagulable state resulting from down regulation of thrombomodulin expression, activation of factor $\mathrm{V}$, inhibition of protein $\mathrm{C}$ activation, and perhaps increased platelet aggregation $^{[11-14]}$. Homocysteine has been linked in numerous in vitro studies with a diversity of mechanisms that could potentiate atherothrombosis, including disrupted endothelial function, impaired protein $\mathrm{C}$ activation, increased thrombin generation, and platelet aggregation. ${ }^{[1-6]}$
Given the relationship between Hcy and thrombosis, a high prevalence of thrombosis would be expected in patients with megaloblasticanemia. As a consequence of hyperhomocysteinemia, patients with acquired vitamin deficiency of vitamin B12/folate had a high risk of thrombosis. However, a more extensive study that controls risk variables and genetic factors is needed to sort out the various contributing factors. ${ }^{[6 \mathrm{~b}]}$

An increase in blood Hcy appears to be associated not only with chronic heart failure but also with acute myocardial infarction (MI). However, although treatment of MI patients with B vitamins achieved a substantial reduction in total plasma Hcy levels, it did not lower the risk of cardiovascular disease recurrence. $^{[5]}$

It was found that the number of patients taking medication associated with hyperhomocysteinemia (such as methotrexate, phenytoin, carbamazepine, or oral contraceptives) or the number who smoked did not significantly increase after acute stroke. ${ }^{[8-9]}$ The risk of MI especially in smokers may at least partly be attributed to hyperhomocysteinemia or low folate. ${ }^{[10]}$

The evolution of homocysteine (Hcy) changes after acute myocardial infarction is still not elucidated. tHcy is elevated in the period predating stroke or $\mathrm{MI}$ and that concentrations temporarily fall in the acute phase by an as yet undetermined mechanism. It has been suggested that this may be related to the acute-phase response, with dilution of tHcy by increased synthesis of plasma proteins.It is still not clear whether the Hcy is a culprit or an innocent bystander in cardiovascular diseases. Addressing the discrepancies in Hcy changes in patients with acute myocardial infarction might give insight in Hcy role in cardiovascular diseases and offer implications both for the clinical interpretation and patients risk stratification. ${ }^{[8-9]}$

Homocysteine level was significantly higher in acute MI in patients without any risk factors and were considered low risk according to the Framingham risk score. The findings support the 
hypothesis that homocysteine level may be an independent risk factor for coronary artery disease. $^{[12-14]}$

Low homocysteine levels in elderly non-vitaminsupplemented hospitalized patients should not be interpreted as a protective factor in some individuals. Instead, it may be considered as an effect of an inflammatory-malnutrition process associated with a poor prognosis. Treatment with B vitamins did not lower the risk of recurrent cardiovascular disease after acute myocardial infarction. A harmful effect from combined B vitamin treatment was suggested. Such treatment should therefore not be recommended. ${ }^{[11][15]}$ Treatment with B vitamins did not lower the risk of recurrent cardiovascular disease after acute myocardial infarction. ${ }^{[11]}$ Widely practiced folic acid fortification in the United States may mask or even worsen vitamin B12 deficiency over time, leading to more severe cases of vitamin B12 deficiency and hyperhomocysteinemia than seen in the past. ${ }^{[25]}$

The Multiple Risk Factor Intervention Trial (MRFIT) also suggested that homocysteine may be a stronger risk factor for the recurrence of events than for a first cardiovascular event.The relationship between plasma homocysteine and prognosis has been less well studied. The reported effect of homocysteine as a prothrombotic factor might lead one to predict that high homocysteine might exacerbate intracoronary thrombosis during the acute phase of these syndromes. In addition, the known effect of high homocysteine on endothelium, seen most dramatically in homocystinuria, might cause a more aggressive course of ischemic heart disease after discharge, leading to more rapid reinfarction and death in the follow-up period. ${ }^{[17-23]}$

In contrast, patients with elevated Hcy levels on the day of the infarction may have a history of coronary atherosclerosis with asymptomatic myocardial ischemia that had already affected their myocardial cells. The subsequent decrease in levels on the days after infarction might be explained by a reduction in the total number of ischemic myocardial cells due to post-MI necrosis. It was previously reported that higher blood Hcy levels in MI patients correlated with worse mid-long-term outcomes. ${ }^{[24]}$

Furthermore, raised homocysteine concentrations are associated with asymptomatic carotid artery wall thickening and stenosis and correlate with the severity of cerebral artery stenosis. It could therefore be postulated that elevated tHcy is a risk factor for atherothrombotic stroke in particular. Second, there is debate about whether tHcy is a causative risk factor in stroke and MI or is merely a secondary marker of risk in survivors. Changes in factors known to affect Hcy metabolism, such as B12 and folate concentrations, smoking habit, and drug history, were also assessed to determine whether these were responsible for any observed change in tHcy concentration observed between the acute and convalescent periods. Increased serum homocysteine is associated with sudden death in the absence of acute coronary thrombosis, especially with concomitant diabetes, and with the presence of lipid-poor, fibrous plaques. Because thrombosis is a component of the progression of atherosclerosis, small increases in total homocysteine may accelerate atherosclerosis by a thrombotic mechanism. However, mechanisms independent of thrombosis may play a role in homocysteine-mediated atherogenesis, and unstable coronary syndromes are not always associated with elevations of total homocysteine. ${ }^{[24]}$

Inverse relationship between serum levels of vitamin B12 and homocysteine in patients. the importance of testing for hyperhomocysteinemia as part of a workup for atherothrombotic disease, especially in patients without other significant risk factors. ${ }^{[25]}$

As conventional risk factors fail to account for part of the cases, homocysteine, a "new" risk factor, is being viewed with mounting interest. Additional risk factors (smoking, arterial hypertension, diabetes, and hyperlipidemia) may additively or, by interacting with homocysteine, synergistically (and hence over proportionally) 
increase overall risk. Supplementation is inexpensive, potentially effective, and devoid of adverse effects and, therefore, has an exceptionally favorable benefit/risk ratio. Folic acid deficiency is considered the most common cause of hyperhomocysteinemia. The results of ongoing randomized controlled intervention trials must be available before screening for and treatment of hyperhomocysteinemia can be recommended for the apparently healthy general population. Most known forms of damage or injury are due to homocysteine-mediated oxidative stresses. Especially when acting as direct or indirect antagonists of cofactors and enzyme activities, numerous agents, drugs, diseases, and life style factors have an impact on homocysteine metabolism. ${ }^{[26]}$

Hyperhomocysteinemia, considered "the cholesterol of nineties", is an established risk factor for cardiovascular diseases and premature atherosclerosis.. More recently, hyperhomocysteinemia was associated with venous thrombosis. Several studies found a correlation with a usual site of thrombosis (central retinal vein, mesenterical level, cerebral veins, Budd-Chiari syndrome). Other studies showed the association between hyperhomocysteinemia and recurrent venous thrombosis. ${ }^{[27]}$

Plasma concentrations of folate and pyridoxal-5'phosphate and folate intake were inversely associated with extracranial carotid stenosis after adjustment for age, sex, and other risk factors. Data indicate a high prevalence of hyperhomocysteinemia in the Framingham Study population, the majority of which can be attributed to vitamin status and that this hyperhomocysteinemia is clinically relevant because of its association with increased risk of occlusive extracranial carotid stenosis. Insufficient levels of folate, and to a lesser extent vitamin B6, appear to predict part of this elevated risk through their role in homocysteine metabolism. These studies also indicate that the recently-implemented fortification of grain and cereal products with folic acid resulted in a substantial decline in plasma homocysteine. ${ }^{[28]}$

Hyperhomocyst(e)inaemia is common in patients with peripheral arterial occlusive disease, coronary heart disease, cerebrovascular disease, carotid artery stenosis and venous thromboembolism. Endothelial dysfunction may be one underlying cause leading to proatherogenic effects associated with hyperhomocyst(e)inaemia. However, the mechanisms which lead to impaired endothelial function in hyperhomocyst(e)inaemia are not fully understood. Recent evidence suggests that homocyst(e)ine may interact with physiological mediators of the endothelial matrix. Oxidative mechanisms and decreased biological activity of endothelium-derived nitric oxide (NO) may also contribute to homocyst(e)ine-associated endothelial dysfunction. B vitamins are essential cofactors in the metabolism of homocyst(e)ine to methionine via the remethylation-pathway (vitamin B12, folic acid) and to cystathionine via the transsulphuration-pathway (vitamin B6). Dietary deficiencies of folic acid, vitamin B12, and vitamin B6 appear to be common among elderly people in the western world and represent one pathogenic factor related to the incidence of hyperhomocyst(e)inaemia. ${ }^{\text {[29] }}$

In patients with chronic hyperhomocyst(e) inaemia, endothelial function is impaired. However, whether hyperhomocyst(e) inaemia per se is a cause or an epiphenomenon of endothelial dysfunction remains unknown. Co-administration of folic acid did not attenuate methionine-induced hyperhomocyst(e) inaemia but completely prevented endothelial dysfunction. Our results suggest that in humans a methionine-rich diet may acutely impair endothelial function, which can be prevented by folic acid supplementation. ${ }^{[30]}$

Since mild homocysteine elevation is easily normalized by B vitamin supplementation, usually with folic acid, it remains for controlled clinical trials of this inexpensive therapy to determine whether normalizing mild homocyst(e)ine elevation reduces cardiovascular risk. ${ }^{[31]}$ 
Simple, inexpensive, nontoxic therapy with folic acid, vitamin B6, and vitamin B12 reduces plasma homocyst(e)ine levels. Although the association between homocyst(e)ine levels and cardiovascular disease is generally strong and biologically plausible, the data from the prospective studies are less consistent. In addition, epidemiologic observations of an association between hyperhomocyst(e) inemia and cardiovascular risk do not prove the existence of a causal relation. Therefore, the effectiveness of folate, vitamin B6, and vitamin B12 in reducing cardiovascular morbidity and mortality requires rigorous testing in randomized clinical trials. Several such trials are under way; their results may greatly affect cardiovascular morbidity and mortality, given the simplicity and low cost of vitamin therapy. ${ }^{[32]}$

In young individuals especially 20-30 years age group who do neither have history of premature CAD in family and no history of HTN/DM/ DYSLIPIDEMIA nor have smoking/alcohol habits a strong suspicion of serum vitamin B12 deficiency and elevated levels of homocysteine should be suspected keeping in mind stressful life styles and junk foods as risk factors.

Public health education about homocysteine and its reduction by increasing supplements of folate and vitamin B12 along with lifestyle changes (diet+exercise+stress management) may reduce the incidence of coronary artery disease as a preventive strategy but not in the treatment of recurrent cardiovascular disease after acute myocardial infarction.

\section{Abbreviations}

total plasma homocysteine $=($ tHcy $)$

\section{References}

1. The predictive value of vitamin $\mathrm{B} 12$ concentrations and hyperhomocyste inaemia for cardiovascular disease M.G.H. van Oijen, R.J.F. Laheij, J.B.M.J. Jansen, and F.W.A. VerheugtNeth Heart J. 2007 Sep; 15(9): 291-294. PMCID: PMC1995101
2. Young myocardial infarction Secondary to vitamin B12 deficiency Shital Rathod1*, Asef Sayyed2, Jayesh Mahale3, Vijay Gaikwad4

3. Serum homocysteine concentration in patients with acute MI and chronic IHD., Banu S1, Mollah FH, Alam MK, Rahman MA, Hamid MA, Wahab MA, Arslan MI.

4. Role of vitamins B6, B12 and folic acid on hyperhomocysteinemia in a Pakistani population of patients with acute myocardial infarction. Iqbal MP1, Ishaq M, Kazmi KA, Yousuf FA, Mehboobali N, Ali SA, Khan AH, Waqar MA.

5. Two models of homocysteinebehavior in acute myocardial infarction. Osorio A1, Ortega E, Ruiz-Requena E.

6. Serum Homocysteine Levels In Patients With Acute Myocardial Infarction In Jordan, AkramSaleh, Nabil Basher

6b. Enhanced risk of thrombotic disease in patients with acquired vitamin B12 and/or folate deficiency: role of hyperhomocysteinemia. Remacha AF1, Souto JC, Rámila E, Perea G, Sarda MP, Fontcuberta J., Ann Hematol. 2002 Nov;81(11):616-21. Epub 2002 Nov 9.

7. Correlation of homocysteine levels with the extent of coronary atherosclerosis in patients with low cardiovascular risk profiles , Wei-Chuan Tsai, MD Yi-Heng Li, MD Liang-Miin Tsai, MD Ting-Hsing Chao, MD Li-Jen Lin, MD Tsai-Yun Chen, MD Jyh-Hong Chen

8. Plasma Homocysteine Concentrations in the Acute and Convalescent Periods of AtherothromboticStroke,D. J. Meiklejohn, MBChB; M. A. Vickers, MD; R. Dijkhuisen, MD;M. Greaves, PhD

9. Changes in serum homocysteine level follow two different trends in patients during early post myocardial infarction period, AminaValjevac, AlenDzubur, EminaNakas-Icindic 
10. Whole blood folate, homocysteine in serum, and risk of first acute myocardial infarction ,B Christensen ,S Landaas,IStensvold,SDjurovic,LRetterst $\varnothing 1$, JRingstad,KBerg,D.SThelle

11. Homocysteine lowering and cardiovascular events after acute myocardial infarction,N. Engl. J. Med., 354 (2006), pp. 1-10 E. Arnesen, H. Refsum, K.H. Bonna, P.M. Ueland, O.H. Forde, J.E. Nordrehaug

12. Serum Vitamin B12 and Folic Acid Levels in Acute Cerebral Atherothrombotic Infarction, Abdulkadir Kocer, NurhanInce, Cuneyt E Canbulat

13. A study of serum homocysteine levels in acute myocardial infarction patients Pooja Sorathia, Ramesh Pradhan and Rosy Lekharu, B.

14. Homocysteine and myocardial infarction, Lars E. Brattström, Björn L. Hultberg

15. Homocysteine Lowering and Cardiovascular Events after Acute Myocardial Infarction; Kaare Harald Bønaa, M.D., Ph.D., Inger Njølstad, M.D., Ph.D., Per Magne Ueland, M.D., Ph.D., Henrik Schirmer, M.D., Ph.D., AageTverdal, Ph.D., TerjeSteigen, M.D., Ph.D., Harald Wang, M.D., Jan Erik Nordrehaug, M.D., Ph.D., EgilArnesen, M.D., and Knut Rasmussen, M.D., Ph.D., for the NORVIT Trial Investigators*N Engl J Med 2006; 354:1578-1588April 13, 2006DOI: 10.1056/NEJMoa055227

16. Homocysteine and myocardial infarction. ,Israelsson B1, Brattström LE, Hultberg BL.

17. Evaluation Of Serum Homocysteine As An Independent Risk Factor For Myocardial Infarction In Young Patients, Asutosh P Chauhan, Piyush B Tailor, Rachit Joshi, PrakashBhabhor

18. Evaluation of serum homocysteine level in acute myocardial infarction patients of Nanded district, Anjali Pergulwar*, A M
Siddiqui , Siddiqui, H N Khan, A R Shinde, S B Khannade.

19. Study of Methionine, Vitamin B12, and Folic Acid Status in Coronary Atherosclerotic Male Patients*M Djalali 1, SR A Hoseiny 1, F Siassi 1, N Fardad 1, R Ghiasvand 1, TR Neyestani

20. A Study Of Serum Homocysteine Levels In Acute Myocardial Infarction Patients, Pooja Sorathia, Ramesh Pradhan and Rosy Lekharu, B.

21. Serum Homocysteine Levels As a Novel Biomarker in Patients with Acute Myocardial Infarction VinodWali*, Singi Yatiraj, Kiran Deshmukh,Nagaraj Bijapur

22. Effect of Plasma Homocysteine Concentration on Early and Late Events in Patients With Acute Coronary Syndromes , Peter J. Stubbs, Mohamed K. AlObaidi, Ronan M. Conroy, MusB, Paul O. Collinson, MRCPath, Ian M. Graham, FRCPI and Mark I. M. Noble

23. Increased Serum Homocysteine and Sudden Death Resulting from Coronary Atherosclerosis With Fibrous Plaques, Allen P. Burke, V. Fonseca, Frank Kolodgie, Arthur Zieske, Louis Fink, RenuVirmani

24. Serum Homocysteine Levels in Patients with Myocardial Infarction in Gorgan (In Northern Iran) Hamid Reza Joshaghani, Ahmad Ali Shirafkan and Abdoljalal Marjani

25. Acute myocardial infarction and pulmonary embolism in a young man with pernicious anemia-induced severe hyperhomocysteinemia. Melhem A1, Desai A, Hofmann MA.

26. Clinical use and rational management of homocysteine, folic acid, and B vitamins in cardiovascular and thrombotic diseases. Stanger O1, Herrmann W, Pietrzik K, Fowler B, Geisel J, Dierkes J, Weger M., Z Kardiol. 2004 Jun;93(6):439-53. 
27. Hyperhomocysteinemia: clinical and therapeutical involvement in venous thrombosis. Hoţoleanu C1, Porojan-Iuga M, Rusu ML, Andercou A., Rom J Intern Med. 2007;45(2):159-64.

28. Relationship between plasma homocysteine and vitamin status in the Framingham study population. Impact of folic acid fortification. Selhub J1, Jacques PF, Bostom AG, Wilson PW, Rosenberg IH., Public Health Rev. 2000;28(1-4):11745.

29. Homocysteine, endothelial dysfunction and cardiovascular risk: pathomechanisms and therapeutic options]. Sydow K1, BögerRH., Z Kardiol. 2001 Jan;90(1):111.

30. Endothelial dysfunction by acute hyperhomocyst(e)inaemia: restoration by folic acid. Usui M1, Matsuoka $\mathrm{H}$, Miyazaki H, Ueda S, Okuda S, Imaizumi T.,ClinSci (Lond). 1999 Mar;96(3):235-9.

31. B vitamins and homocysteine in cardiovascular disease and aging. Wilcken DE1, Wilcken B., Ann N Y Acad Sci. 1998 Nov 20;854:361-70.

32. Homocyst(e)ine and cardiovascular disease: a critical review of the epidemiologic evidence. Eikelboom JW1, Lonn E, Genest J Jr, Hankey G, Yusuf., Ann Intern Med. 1999 Sep 7;131(5):36375. 